\title{
Zeolitic Imidazolate Framework-Derived Nanoarchitectures for Lithium Metal Storage Medium
}

\author{
Jaewoo Lee, ${ }^{a}$ Sang A Han, ${ }^{a}$ Hamzeh Qutaish, ${ }^{a}$ Lok Kumar Shrestha, ${ }^{b}$ Katsuhiko Ariga, ${ }^{b, c}$ and Jung Ho Kim ${ }^{\star, a}$ \\ ${ }^{a}$ Australian Institute for Innovative Materials, University of Wollongong, NSW 2500, Australia \\ ${ }^{b}$ National Institute for Materials Science (NIMS), 1-1 Namiki, Tsukuba 305-0044, Japan \\ ${ }^{c}$ Department of Advanced Materials Science, Graduate School of Frontier Sciences, The University of Tokyo, \\ 5-1-5 Kashiwanoha, Kashiwa, Chiba 277-8561, Japan
}

Email: jhk@uow.edu.au (J. H. K.)

\begin{abstract}
Since metal-organic frameworks were firstly suggested and developed as highly porous materials, zeolitic imidazolate framework (ZIF) derived carbons have been considered as promising materials for energy conversion/storage applications. In particular, ZIF-derived carbons are among the most feasible media in which to store metallic lithium ( $\mathrm{Li}$ ) to create a practical lithium-ion battery anode, because of their large pore volume and easily adjustable properties. With this background, we suggest that the electrochemical performance of this Li-metal storage medium can be maximized by controlling the properties of ZIF-derived carbon. Finally, we discuss perspectives on future Li-metal anode research.
\end{abstract}

Keywords lithium-metal anode, metal-organic frameworks, porous carbon, zeolitic imidazolate frameworks

Metal-organic frameworks (MOFs) have received great attention as attractive porous materials due to their high surface area, controllable pore size/volume, and facile synthesis. ${ }^{[1-3]}$ Historically, MOFs or porous coordination polymers (PCPs) were suggested and developed by Yaghi's ${ }^{[4]}$ and Kitagawa's ${ }^{[5]}$ groups based on zeolite chemistry. Among the MOF subclasses, an MOF- 5 was found in 1999 to be the first ideal structure, with thermal, mechanical, and chemical stability for various applications. ${ }^{[6]}$ Owing to the insulating nature of MOFs, however, there are still drawbacks for energy conversion/storage applications. In recent years, hybridized MOF-derived nanostructures with conducting polymer or various carbons have been suggested as alternative next-generation electrodes. ${ }^{[7]}$ Considering this background, MOF-derived porous carbon structures were successfully obtained by heating a secondary carbon precursor within the pores of the MOF. Later, Yamauchi's ${ }^{[8]}$ and Park's ${ }^{[9]}$ groups reported a straightforward way to make hierarchically porous carbons without a secondary carbon precursor. These have gained more attention because they allow a direct carbonaceous approach to the zeolitic imidazolate framework (ZIF) series, which are one subclass in the MOF family. Since then, ZIF-derived materials have been considered for energy storage applications. ${ }^{[10-14]}$

Representative examples are ZIF-8 and ZIF-67, which are composed of $\mathrm{Zn}^{2+}$ and $\mathrm{Co}^{2+}$ metal ions, respectively. Both are also strongly coordinated by 2-methylimidazole (2-MIM) as the organic linker. These ZIFs are formed as rhombic dodecahedra with a large cavity $(11.6 \AA) .{ }^{[15]}$ From these two types of ZIFs, typical nanoporous carbon can be obtained through chemical etching and calcination. ZIF-8 derived carbon is in an amorphous phase containing mainly micropores, and it has a relatively high nitrogen content and large surface area. On the other hand, ZIF-67 has a highly-crystalline carbon structure with mainly mesopores, relatively low nitrogen content, and a small surface area. ${ }^{[16]}$ The Co particles are the key factor required to grow carbon nanotubes (CNTs). ${ }^{[17]}$ Very interestingly, a $\mathrm{Co}^{2+}$ excess hybrid ZIF $\left(\mathrm{Co}^{2+}: \mathrm{Zn}^{2+}=2: 1\right)$ generates more CNT growth, which results in enhanced electrical conductivity, even if bare ZIF-67 derived carbons have more residual Co particles in their carbon structure. ${ }^{[12,16,17]} \mathrm{We}$ argue that this hybrid ZIF offers a synergetic effect from its two metal sources. Very recently, ZIF-8 nanobubbles were converted into hollow carbon through tannic acid solution without any degradation of their features. ${ }^{[18]}$ Figure 1 is an illustration of various ZIF-derived carbons, which are originally derived from different kinds of ZIF crystals, single ZIFs (Zn, Co), bimetallic ZIFs (Zn/Co hybrid), and hollow ZIFs (tannic acid etching). For practical energy storage applications, it is necessary to control their surface area/pore volume, particle size/shape, monodispersity, and element doping.

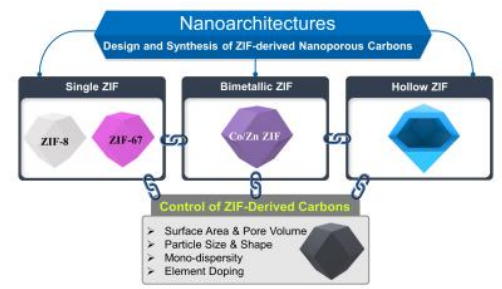

Figure 1 Illustration of various types of ZIF crystals with their carbonized forms.

Based on our results regarding the carbons derived from ZIF-8, ZIF-67, and bimetallic ZIF, each of these properties can be comfirmed and compared, ${ }^{[16,17]}$ as shown in Table 1.

Recently, the development of Li-metal storage anodes is under the spotlight among Li-ion battery researchers because it provides much higher energy density compared to the conventional graphite-based anode system. ${ }^{[19]}$ The prevention of $\mathrm{Li}$ dendritic growth, however, as well as a facile synthesis for reducing production costs, should be considered when exploring an appropriate Li-metal storage medium. In this respect, ZIF-derived carbons could pave the way towards the practical application of Li-metal anodes, due to their large pore volume 
Table 1 Intrinsic properties of each type of ZIF-derived carbon

\begin{tabular}{|c|c|c|c|}
\hline Properties & ZIF-8-C & ZIF-67-C & Bimetallic ZIF-C \\
\hline Surface area $/\left(\mathrm{m}^{2} \mathrm{~g}^{-1}\right)$ & 765 & 354 & 432 \\
\hline Pore volume $/\left(\mathrm{cm}^{3} \mathrm{~g}^{-1}\right)$ & 0.45 & 0.38 & 0.55 \\
\hline Pore size & micropores & mesopores & micropores/mesopores \\
\hline Particle size $/ \mathrm{nm}$ & 500 & 1200 & 500 \\
\hline Electrical conductivity/( $\left.\mathrm{S} \mathrm{cm}^{-1}\right)$ & $<0.1$ & $\sim 10$ & $>10$ \\
\hline
\end{tabular}

${ }^{a}$ Controllable by simple change of synthetic conditions.

and easy adjustable properties. ${ }^{[1]}$

Meanwhile, the theoretical volumetric capacity of Li-metal is $2062 \mathrm{mAh} \mathrm{cm}^{-3}\left(3861 \mathrm{mAh} \mathrm{g}^{-1} \times 0.534 \mathrm{~g} \mathrm{~cm}^{-3}\right) .{ }^{[20]}$ Based on this, the theoretical gravimetric capacity of a ZIF-derived carbon, when it reaches full capacity for storing Li-metal in its pores, including Li-ion intercalation into carbon, can be calculated by the simple equation below:

Gravimetric capacity of a ZIF-derived carbon $\left(\mathrm{mAh} \mathrm{g}^{-1}\right)=$ $\left[2062\left(\mathrm{mAh} \mathrm{cm}^{-3}\right) \times\right.$ pore volume $\left.\left(\mathrm{cm}^{3} \mathrm{~g}^{-1}\right)\right]+372\left(\mathrm{mAh} \mathrm{g}^{-1}\right)(1)$

As shown in Figure 2, the calculated theoretical capacities of ZIF-67-C, ZIF-8-C, and bimetallic ZIF-C are 1156, 1300, and $1506 \mathrm{mAh} \mathrm{g}^{-1}$, respectively. In addition, the theoretical gravimetric capacity of hollow ZIF-carbon can be estimated by finding the macroporous hollow volume and dividing it by the total volume of the material. In previous studies, hollow ZIF-carbons contained approximately $20 \%$ to $50 \%$ hollow macropore volume. ${ }^{[18,21]}$ Thus, they had 0.7 to $1.1 \mathrm{~cm}^{3} \mathrm{~g}^{-1}$ of total pore volume, including micro-, meso-, and even macropore volume, and consequently, the hollow ZIF-carbon showed higher capacity than the other types of ZIF-derived carbons.

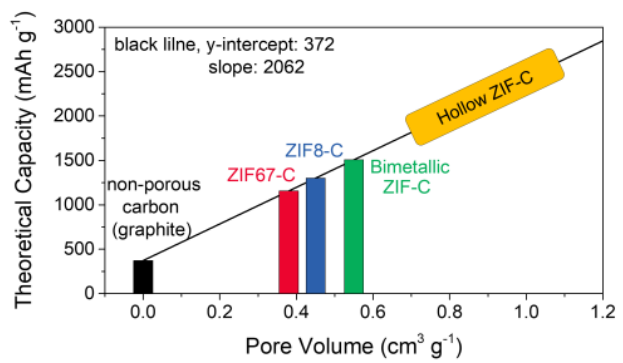

Figure 2 Theoretical capacities of each type of ZIF-derived carbon.

Nevertheless, the adequacy of its electrical conductivity for inducing a smooth electrochemical reaction is not guaranteed, and its etching process with consequent low production yield will decrease the production efficiency. On the other hands, bimetallic ZIF-carbon shows high electrical conductivity with reasonable pore volume, as well as having a relatively facile synthetic process. For this reason, it is necessary to consider every relevant factor comprehensively, when exploring the optimum ZIF-carbon Li-metal storage medium. To sum up, in order to discover ZIF-derived carbons that are suitable Li-metal storage media, i) large pore volume, ii) suitable pore size, iii) acceptable electrical conductivity, iv) cost effectiveness, and v) particle size and surface area that are fit for purpose should be considered concurrently.

From this aspect, we are optimistic about the development of an advanced Li-metal storage medium through devoting research efforts to studying the ZIF-derived carbons in the future. Especially, we prospect the bimetallic ZIF-derived carbon with higher electrical conductivity and production yield would provide reasonable outcomes on the development of

\section{Li-metal anode batteries.}

\section{Acknowledgements}

This research was supported by the Technology Development Program to Solve Climate Change through the National Research Foundation of Korea (NRF), funded by the Ministry of Science and Information and Communications Technology (ICT) (NRF-2018M1A2A2063347) and UOW-MANA project.

\section{References}

[1] Furukawa, H.; Cordova, K. E.; O’keeffe, M.; Yaghi, O. M. Science 2013, 341, 1230444

[2] Zhao, J.; Liu, X.; Wu, Y.; Li, D. -S.; Zhang, Q. Coord. Chem. Rev. 2019, 391, 30.

[3] Li, P.; Cheng, F. -F.; Xiong, W. -W.; Zhang, Q. Inorg. Chem. Front. 2018, 5, 2693.

[4] Eddaoudi, M.; Kim, J.; Rosi, N.; Vodak, D.; Wachter, J.; O'keeffe, M.; Yaghi, O. M. Science 2002, 295, 469.

[5] Susumu, K.; Mitsuru, K. Bull. Chem. Soc. Jpn. 1998, 71, 1739.

[6] Li, H.; Eddaoudi, M.; O'keeffe, M.; Yaghi, O. M. Nature 1999, 402, 276.

[7] Kim, J.; Kim, J. H.; Ariga, K. Joule 2017, 1, 739.

[8] Chaikittisilp, W.; Ariga, K.; Yamauchi, Y. J. Mater. Chem. A 2013, 1, 14.

[9] Yang, S. J.; Lim, T.; Im, J. H.; Kim, Y. S.; Lee, K.; Jung, H.; Park, C. R. Chem. Mater. 2012, 24, 464.

[10] Salunkhe, R. R.; Kaneti, Y. V.; Kim, J.; Kim, J. H.; Yamauchi, Y. Acc. Chem. Res. 2016, 49, 2796.

[11] Salunkhe, R. R.; Tang, J.; Kobayashi, N.; Kim, J.; Ide, Y.; Tominaka, S.; Kim, J. H.; Yamauchi, Y. Chem. Sci. 2016, 9, 5704.

[12] Kim, J.; Young, C.; Lee, J.; Heo, Y. -U.; Park, M. -S.; Hossain, Md. S. A.; Yamauchi, Y.; Kim, J. H. J. Mater. Chem. A 2017, 5, 15065.

[13] Khan, A. H.; Ghosh, S.; Pradhan, B.; Dalui, A.; Shrestha, L. K.; Acharya, S.; Ariga, K. Bull. Chem. Soc. Jpn. 2017, 90, 627.

[14] Wu, Z.; Xie, J.; Xu, Z. J.; Zhang, S.; Zhang, Q. J. Mater. Chem. A 2019, 7, 4259.

[15] Tang, J.; Salunkhe, R. R.; Zhang, H.; Malgras, V.; Ahamad, T.; Alshehri, A. M.; Kobayashi, N.; Tominaka, S.; Ide, Y.; Kim, J. H.; Yamauchi, Y. Sci. Rep. 2016, 6, 30295.

[16] Han, S. A; Lee, J.; Shim, K.; Lin, J.; Shahabuddin, M.; Lee, J. -W.; Kim, S. -W; Park. M. -S.; Kim, J. H. Bull. Chem. Soc. Jpn. 2018, 91, 1474.

[17] Kim, J.; Young, C.; Lee, J.; Park, M. -S.; Shahabuddin, M.; Yamauchi, Y.; Kim, J. H. Chem. Commun. 2016, 52, 13016.

[18] Zhang, W.; Jiang, X.; Zhao, Y.; Carné-Sánchez, A.; Malgras, V.; Kim, J.; Kim, J. H.; Wang, S.; Liu, J.; Jiang, J. -S.; Yamauchi, Y.; Hu, M. Chem. Sci. 2017, 8, 3538.

[19] Lin, D.; Liu, Y.; Cui, Y. Nat. Nanotechnol. 2017, 12, 194.

[20] Li, S.; Jiang, M.; Xie, Y.; Xu, H.; Jia, J.; Li, J. Adv. Mater. 2018, 30, 1706375.

[21] Yang, D. -H.; Zhou, H. -Y; Liu, H.; Han, B. -H. iScience 2019, 13, 243.

Received June 24, 2019 Accepted July 30, 2019 